\title{
A Hardware Design of Vehicle Information Platform
}

\author{
YingqingWu \\ College of Mechatronics Engineering and Automation, \\ Shanghai University, \\ Shanghai 200072, China
}

\begin{abstract}
Mobile devices have been widely used to assist the existing public transportation system. A new solution to the vehicle information collection is introduced. The hardware design of a vehicle information communication platform is mainly introduces, including platform power distribution design, the battery design, UART port expansion, the power supply isolation design and impedance matching design. Part of the final hardware platform debugging result is proposed. After testing, each power reduction module works stably and the GPS signal receiving ability is great.
\end{abstract}

Keywords-vehicle information platform; GPS; embedded; ARM.

\section{INTRODUCTION}

Nowadays the bus has a variety of equipment to assist the operation of intelligent public transit system. But the vehicle equipment at present still has some problems. For example, almost all information terminals on the bus are custom-made that lack universality and cannot update with the development of surrounding vehicle equipment; GPS vehicle equipment can give the location of public transit vehicle but cannot communicate with the station reporting machine and is unable to achieve full automatic stop function that it will cause the driver take the operating condition of the station reporting machine into account in the driving process. If you want to reduce the driver's burden, the blindness of operation to a great extent and reduce driver fatigue phenomenon to improve the traffic safety, there should be a change in the existing technical scheme and system structure from the fundamental including information acquisition, information exchange, driving and location, vehicle control, automobile security and so on to make the car more humanized, comfortable and individual [1]. So in this paper, the embedded technology is used to form a new information exchange platform to improve the existing vehicle equipment operation.

\section{THE SYSTEM HARDWARE ARCHITECTURE DESIGN}

Consider the vehicle equipment nowadays has become increasingly diversified and functional requirements become more and more complicated, it needs to differentiate the vehicle equipment according to the functional requirements on the hardware. The core information exchange processing function, extra integrated CDMA and CPS, vehicle flow record and other related functions are realized by the same hardware terminal. However the present mature bus card machine, station report machine and other equipment are plug in the core information exchange in a device independent way. The specific connection method is shown in figure 1 .

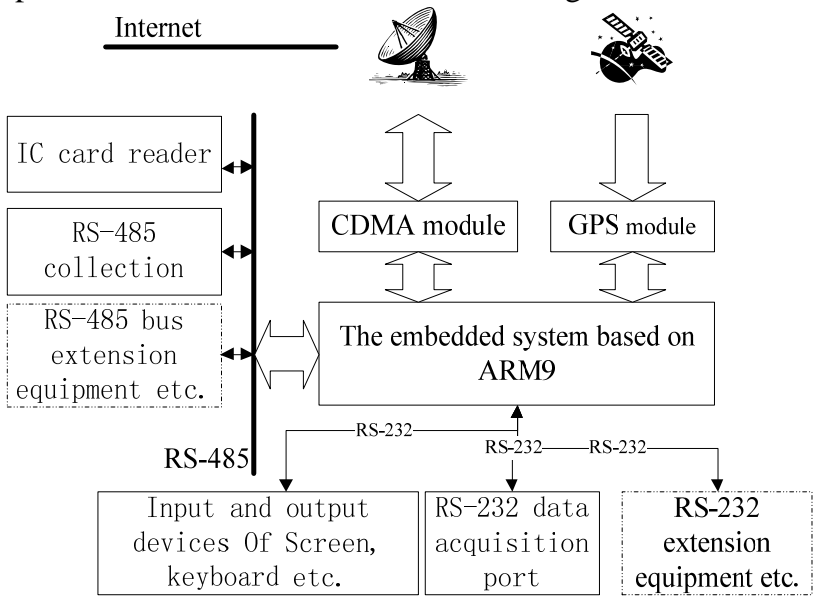

Figure 1. system structure design based on the vehicle information communication platform

In consideration of the constraint on the bus running bump, limited power supply, car space constraint and other factors, the solution using PC technology is clearly not realistic. In contrast, the embedded system has unique advantages of low power consumption, small occupation of resources, high integration, high performance and strong stability. The use of embedded technology for researching and developing vehicle terminal is the best solution. This design uses ARM embedded system architecture, integrates CDMA module and GPS module to provide a powerful platform for realizing intelligent transportation system (ITS).

The vehicle information communication platform adopts ARM structure to design. The S3C2410A220 processor based on ARM920T kernel produced by SAMSUN G Company is selected. The processor's frequency is up to $203 \mathrm{MHz}$ that can meet the requirement as the main controller of vehicle information communication platform. It has rich resources on chip [2]. Using these resources, plus two SDRAM chips K4S281632I UC/ 75 with capacity of 32M and one NAND Flash chip K9F1208UOC PIB0 with capacity of $32 \mathrm{M}$, the minimum system that meet the system function demand can be built

The CDMA module uses Huawei Em200. This module is a communication module working in the CDMA800M band .It has wide temperature range from -20 degrees 
Celsius to 70 degrees Celsius [3]. The vehicle communication platform is integrated with that module. The platform can communicate through the serial asynchronous communication and control through wireless module AT command.

The GPS module uses U-Blox Neo25Q which is a multifunctional independent GPS module. Its package volume is only $12 \times 16 \times 214 \mathrm{~mm}$. It is based on the ROM architecture without using high cost Flash EPROM so it can achieve wide application requirements of low cost and ultra-small size. And the module has many characteristics and very flexible connection technology selections.Vehicle communication platform also integrates the module, communicates through the serial asynchronous communication mode, and uses the NMEA protocol [1].

The structure of hardware platform is shown in figure 2 .

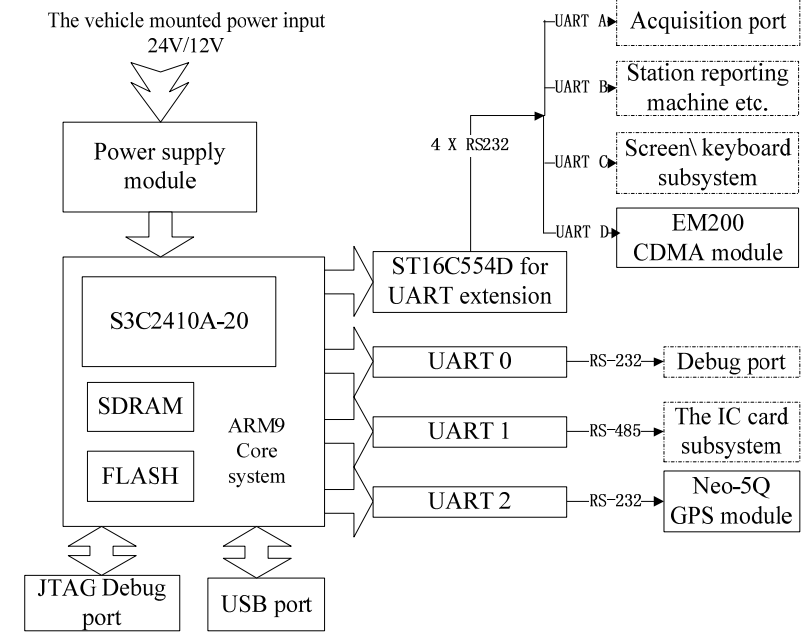

Figure 2. Vehicle information transceiver hardware platform structure

\section{A. The power supply}

The vehicle power supply comes from the car battery while the car battery is greatly affected by automobile engine, especially when launch the vehicle, the power supply input may under voltage that cause the information platform device power off and restart. So it is necessary to add extra power module to let the communication platform work stably. At the same time, the vehicle equipment operation environment is extremely harsh and the power quality is worse than that of the indoor operation device. The large temperature difference between north and south area of our country should also be given full consideration. All devices on the platform must have wide temperature tolerance, at least from 20 degrees Celsius to 70 degrees Celsius. Meanwhile, the needs of the present vehicle application module voltage are different, such as S3C2410AL needs 313V, CDMA module needs 410V, USB device needs $510 \mathrm{~V}$ and so on. Some chip's power supply demand is high, such as the GPS module needs independent LDO to supply power. So in the design of hardware platform, all these factors should be fully taken into account to reasonably select chip devices, distribute power supply and arrange layout of PCB.

\section{B. Planning and design of charging batteryhardware circuit}

In the car platform, 3 kind of devices need backup battery to supply power, include real-time clock power supply to S3C2410AL processor chip, power supply to GPS and to CDMA module.

Real time clockunitRTC S3C2410AL can work when the system power is off relied on the backup battery. The RTCVDD voltage of S3C2410A should be in the range of $118 \mathrm{~V}$ to the highest $217 \mathrm{~V}$. In the test, when the voltage was higher than $217 \mathrm{~V}$, real time clock chip module failed completely, but the chip other function was still running.

For the GPS module, when the main power failure, there should be a backup battery continuous power supply through its $\mathrm{V}_{\text {_ }}$ BCKP pin in order to maintain the real-time clock and the RAM backup. And this is very important, because of the RAM data back, Neo25Q (GPS receiving module) can realize hot start or warm start after power off. The warm start and hot start can maintain part of the module information set (including the ephemeris information that needed long time to acquire and calculation). Using warm start or hot start depends on the time of main power loss. If the backup battery power fails when the main power supply fail, the GPS receiver can only do a longer time cold start. The module's hot start time is less than one second, warm start time is less than half a minute. But if it is a cold start, the time will be long about one minute or so [4-5].

For EM200(CDMA module),its working voltage range of VDD2RTC pin is $214 \sim 412 \mathrm{~V}$ (2) . The EM200 Huawei CDMA module pin of VDD2RTC can supply power for the rechargeable battery whose battery voltage range is $210 \sim$ $510 \mathrm{~V}$.

In the selection of rechargeable batteries, I choose the micro button battery L IR1229 which has been widely used in the electronic area. The battery is a rechargeable Lithium battery and the nominal voltage is $316 \mathrm{~V}$. In the test, the voltage of new brought battery is $4.10 \mathrm{~V}$ or so. Therefore, the voltage of the battery is higher than the working voltage of S3C2410ALand Neo25Q. So it is necessary to reduce the voltage before access to the platform module. Considering the cost and space, 1N4148 Schottky diode is chosen to reduce voltage because of the low cost and small size.

$1 \mathrm{~N} 4148$ is a kind of commonly used high speed Schottky diode. Its positive break-over voltage changes with the temperature of pin node and the forward current. When the node temperature is 25 degree Celsius, the forward break-over voltage is about $0.65 \mathrm{~V}$. The certain positive voltage drop characteristics of Schottky diode is full used to reduce the L IR1220 voltage. Either S3C2410AL, Neo25Q or EM200 module has small current consumption, so the pressure drop in the Schottky diodes is very small. In the test, the pressure drop in each diode is about $0.35 \mathrm{~V}$

This design can guarantee the EM200 module charging on the L IR1220 button battery in a main power supply case that can greatly enhance the endurance of the button battery for on board device. And when the main power is moved, 
the button battery can supply power for the 3 kinds of device to maintain the real-time clock module and related modules of each device.

\section{THE UART EXPANSION METHOD}

CDMA module and GPS module in this design use serial port as the communication interface. But S3C2410AL only has 2 serial ports and a certain amount of serial ports is also needed when external equipment is connected. So the serial port of the vehicle communication platform should be expanded.

There are many kinds of way to expand serial port at present. The more common ways are parallel to serial mode, division multiplexing serial port expansion mode and USB to serial port mode.

Considering the characteristics of various equipment is not confirmed, every serial port leaded needs certain communication ability. So the use of GM8125 chip to expand serial port in a way of time division multiplexing is not desirable. Because time division multiplexing method will reduce the serial communication rate, thereby reducing the performance of serial port communication. However, if using the USB serial expansion method, such as the use of FT2232C chip, it will occupy the USB port of S3C2410AL chip and the driving support for embedded operating system is not perfect. So this method should not be used. In summary, the information communication platform uses ST16C554D chip to expand serial port.

\section{A. Digital optocoupler isolation}

Vehicle information communication platform usually needs to communicate with other various types of vehicle equipment. And if the platform signal is directly connected with the external signal, it will cause the unexpected situation, such as the power fluctuation caused by external equipment, generating interference signal etc., and make the platform to stop working. Such errors tend to make the reliability, stability of system deviation, system halted easily, wrong operation failure and may cause serious damage to system. So it is very effective toisolate the communication platform from external device. Usually the optocoupler isolation method is used.

But there are many disadvantages: unstable, performance is affected greatly by temperature and time, the degree of integration is not high, the size is to large, external device should be connected, etc.. In the use of the high speed photoelectric conversion occasion, high speed optocoupler such as 6N137 is tend to be used usually. But at this time, we chose ADuM1201digital optocoupler of ADI Company. This kind of optocoupler has greatly improved the degree of integration and various aspects of performance compared with the former. Compared with ordinary high speed optocoupler, ADuM has advantages of high speed, low power consumption, high performance, small size, high integration, cheap and flexible application.

The ADuM1201ARZ digital optocoupler chip is a typical product of the ADuM series of high performance digital isolator. Each of the chip has two different directions of the communication channel. I use this chip to isolate the communication of RS485 and RS232. The specific connection way please see figure 3 RS485 electrical isolation.

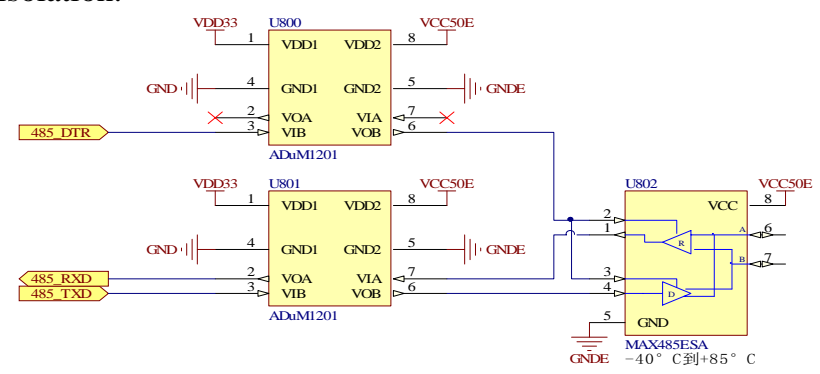

Figure 3. RS-485 Electrical isolation

\section{B. The impedance matching design of GPS module Neo25Qand antenna}

In the design of Neo25Q( a GPS receiving module), 50 ohms impedance matching should be done between GPS antenna and antenna signal line which connect the GPS receiving module to the MMCX antenna interface. The line's impedance matching is very important that it will affect the ability of receiving GPS signal. The traditional impedance design method is through experience formula and looking on the chart to determine the relevant design parameters. The process is complex and cumbersome. Therefore, I choose to use the EDA software AppCAD designed by Agilent Co to calculate impedance. In the design of this coplanar wave line, input line's length, wide, material dielectric constant, the interlayer distance, distance between lines, signal frequency and other specific design parameters, then the impedance of the line can be calculated. By calculation, the line's impedance is 50.7 ohms.

After printing out the actual PCB, the impedance test is done to antenna signal line of Neo25Q. The impedance is 52 ohms which is very close to the theoretical 50.7 ohms. The reason of the error is in many aspects, especially the large error of the material's dielectric constant.

\section{THE DEBUGGING AND RUNNING RESULT}

\section{A. Reduce voltage to LIR1220 by 1 N4147}

After testing, the new rechargeable battery's highest voltage is $4.10 \mathrm{~V}$ and then the input voltage of Neo25Q (GPS receiving module) is $3.38 \mathrm{~V}$ after two voltage decrease processes of $1 \mathrm{~N} 4147$. That is consistent with the $1.4 \sim 3.8 \mathrm{~V}$ working voltage range. At the same time, after 4 voltage decrease processes of $1 \mathrm{~N} 4147$, the input voltage of S3C2410AL is $2.64 \mathrm{~V}$ which is consistent with the $1.8 \sim 2.7 \mathrm{~V}$ working voltage range. In the use process, the battery voltage is gradually decreased to below $4 \mathrm{~V}$.

\section{B. Power consumption (voltage24V)}

After the test, we can see that the minimum system(The minimum system includes a S3C2410AL processor chip, two memory chip and a NAND Flash memory chip and the peripheral part of crystal oscillator circuit, etc.) power consumption is about 60mA (24V input). Noe25Q power consumption is about $50 \mathrm{~mA}$ (24V input) without 
connecting GPS antenna. Power consumption of EM200 HUAWEI CDMA communication module is about $16 \mathrm{~mA}$ (24V input) without SIM card.

\section{The operation situation of GPS module}

In a thunderstorm weather, the GPSantenna was put on the windowsill. Neo25Q uploaded the NMEA protocol information. And U2Center6101 software was used to analytical and graphical display output the information.

\section{SUMMARY}

The vehicle information communication platform has good performance and each of the module works coordinately. The common used vehicle device functions at present are realized such as IC card reader, station reporting machine etc.. The platform provides 3 RS232 interface and 1 RS485 interface altogether. At the same time, the platform provides the GPS location function and CDMA communication function that can realize functions of automatic station reporting and flow information upload to dispatching station. That will be convenient for the bus driver and has a good market prospect.

\section{ACKNOWLEDGEMENTS}

The research work was supported by Shanghai Municipal Science and Technology Commission Fundunder Grant No. 13111102500

\section{REFERENCES}

[1] Xu Zhongming, He Yansong: Study Trend of Foreign Intelligent Transportation System. AUTO INDUSTRY RESEARCH. Forum Vol. 1 (2001), p.34-38

[2] Wu Minghui: The development and application of embedded system based on ARM,edtied by People's Posts and Telecommunications Press (2004)

[3] Huawei: EM200 CDMA 1X module product description .

[4] Lv Fang, Zhang yue, Yang Hongye:GPS Taxi Dispatch System of TsingHua Embedded.Computer Automated Measurement \& Control, Forum Vol. 10(2003),p. 751-753.

[5] Ma Haibo, Chen Yangzhou, Cuipingyuan:Development of Embedded Application Software for Vehicle Navigation System. Computer Measurement \& Control, Forum Vol. 2(2007), p. 188-190. 